\title{
Psychiatrie - forfaits journaliers dégressifs vs forfaits par cas
}

\author{
Jürg Unger-Köppel \\ Dr méd., membre du Comité central de la FMH, responsable du département Médecine et tarifs hospitaliers
}

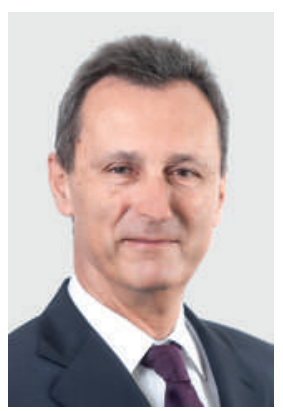

Pourquoi changer ce qui fonctionne encore très bien? La question peut se comprendre, car du point de vue des fournisseurs de prestations, les forfaits journaliers ont fait leurs preuves. Mais la loi spécifie: «Pour rémunérer le traitement hospitalier [...] les parties à une convention conviennent de forfaits. En règle générale, il s'agit de forfaits par cas.»

Lorsqu'il a été question "d'importer les DRG en Suisse», nous avons très rapidement rappelé dans un papier de position de la FMH que les DRG n'étaient pertinents ni pour la psychiatrie ni pour la réadaptation. Ce point de vue ne nous a pas quittés jusqu'à aujourd'hui et le déve-

Les forfaits par cas ne sont pas adaptés à la psychiatrie. La FMH a plaidé avec succès pour un tarif avec des forfaits journaliers dégressifs.

loppement de TARPSY, un tarif psychiatrique sans aucun forfait par cas, représente un succès important pour la FMH. Le tarif essaie de prendre en compte les coûts de traitement de manière équitable avec des forfaits journaliers dégressifs, les travaux s'étant basés sur les chiffres des années 2014 et 2015. Ce genre de système engendrera toujours des cas qui rapportent davantage qu'ils ne coûtent, et vice-versa. Alors, pour que les cliniques puissent s'y préparer, SwissDRG a mis un groupeur à leur disposition à la mi-octobre 2016 leur permettant d'analyser leurs données. Elles pourront ensuite mettre en place les éventuelles mesures nécessaires avant l'introduction du tarif au $1^{\mathrm{er}}$ janvier 2018. Plus le nombre de données sur les différents groupes de patients est restreint, plus le tarif est imprécis. Il faut donc s'attendre à des ajustements supplémentaires, notamment pour la psychiatrie gériatrique et la psychiatrie de l'addiction. S'agissant des enfants et des adolescents, TARPSY présente encore quelques défauts: l'estimation du degré de gravité n'a pas encore été vérifiée avec les données de ces catégories d'âge. La représentativité des enfants est insuffisante. Pour les adolescents, les coûts de séjour sont encore répartis entre des structures pour adolescents et pour adultes, créant ainsi de mauvais incitatifs, car les coûts pour les jeunes sont plus bas dans les cliniques pour adultes. De plus, conformément à la Convention relative aux droits de l'enfant de l'ONU, les adolescents n'ont pas le droit d'être traités dans des unités pour adultes. Par conséquent, TARPSY ne pourra être obligatoire pour les mineurs qu'après les adaptations requises.

Les assureurs souhaitent que la poursuite du développement de TARPSY vise une représentativité la plus précise possible de chaque activité afin de permettre un meilleur contrôle. Or cela ne fait qu'augmenter les tâches administratives des fournisseurs de prestations, tout en diminuant le temps et les ressources consacrés aux patients. Dans ce contexte, il sera primordial de garder une juste vision des choses au cours des années qui viennent pour préserver le bon équilibre entre ces exigences contradictoires, faute de quoi les tâches administratives risquent d'étouffer le système.

En introduisant les DRG après les avoir adaptés et helvétisés, la Suisse a mis en application un système établi depuis plusieurs années dans d'autres pays. TARPSY est en revanche un pur produit «Swiss made». Son premier développement a été initié par la ZHAW à Winterthour et SwissDRG SA a ensuite donné sa forme actuelle au tarif. Ne pas détecter les effets collatéraux indésirables ou les "erreurs de fabrication" qui se seraient glissées en cours de développement reviendrait à les mettre en place à long terme. C'est pourquoi la FMH recommande de prendre un deuxième avis et de consulter un institut indépendant spécialisé dans l'économie de la santé pour analyser le tarif TARPSY. Label «Swiss made» oblige.

TARPSY est une première internationale et, par conséquent, il mérite un deuxième avis. Label «Swiss made» oblige.

Tenons-nous prêts, car le changement arrive. Vous en apprendrez davantage sur TARPSY à la page 1404 de ce numéro du Bulletin des médecins suisses.

Quant au tarif ST Reha développé pour la réadaptation, il n'est pas encore finalisé. Mais les cliniques pourront procéder à de premières projections grâce à une préversion disponible à partir du $1^{\text {er }}$ janvier 2018 et se préparer à l'introduction prévue au $1^{\text {er }}$ janvier 2020. 